\title{
La responsabilidad de proteger: la perspectiva latinoamericana
}

\section{The responsibility to protect: the latin american perspective}

\author{
Ricardo Arredondo ${ }^{1}$ \\ Universidad de Buenos Aires (Argentina)
}

Recibido: 30-03-14

Aprobado: 15-04-14

\section{Resumen}

El artículo trata de la responsabilidad de proteger $(\mathrm{RdP})$, que plantea un dilema entre el respeto a los principios de soberanía y no intervención y la necesidad de proteger a las poblaciones en peligro en casos de violaciones graves, masivas y sistemáticas de sus derechos humanos y/o del derecho internacional humanitario.

En ese contexto, el propósito de este trabajo es analizar las posturas de América Latina sobre la RdP. Sin embargo, cualquier debate sobre esta cuestión debe empezar por considerar brevemente de qué estamos hablando cuando decimos "responsabilidad de proteger", a qué se refiere este principio, qué aspectos abarca y cuál es la situación actual, en relación con el contexto histórico y político que ha determinado muchas de las posturas adoptadas por los países de América Latina al momento de tener que aplicar decisiones de política exterior o fijar sus principios.

Palabras-clave: Responsabilidad de proteger; principio de soberanía y de no intervención; derechos humanos; derecho internacional humanitario; América Latina.

\footnotetext{
${ }^{1}$ (arredondo.ricardo@gmail.com) Abogado (UNT), LLM (LSE), Doctor en Derecho (UBA). Profesor de Derecho Internacional Público, Facultad de Derecho, Universidad de Buenos Aires (UBA), Argentina. Autor de 2012. Intervención humanitaria y responsabilidad de proteger ¿Hacia un nuevo paradigma de protección de los derechos humanos? Buenos Aires: Catálogos; y Director del 2012. Manual de Derecho Internacional Público. La Ley - Thomson Reuters.
} 


\begin{abstract}
The article deals with the responsibility to protect (RtP), which poses a dilemma between respect for sovereignty and non-intervention principles and the need to protect populations at risk for serious, massive and systematic violations of their human rights and / or international humanitarian law.

In this context, the purpose of this paper is to analyze the positions of Latin America on the RtP. However, any discussion of this issue must begin by considering briefly what we mean when we say "responsibility to protect", which refers to this principle, which aspects covers and what is the current situation in relation to the historical and political context has determined that many of the positions taken by the countries of Latin America at the time of having to implement foreign policy decisions or fix her principles.
\end{abstract}

Key-words: Responsibility to protect, sovereignty and non-intervention principles, human Rights, International humanitarian law, Latin America.

\title{
1. Introducción
}

Hasta que el terrorismo acaparó la atención internacional después del 11 de septiembre de 2001, el debate que había dominado la agenda de las relaciones internacionales durante la última década del siglo XX y los primeros años del XXI fue el de la intervención humanitaria, esto es, la cuestión de cuándo, si correspondiera, resulta apropiado que los Estados utilicen la fuerza armada en otro Estado, con el propósito de proteger a las poblaciones vulnerables que se encuentran en peligro en ese otro Estado, como en los casos de Somalia, Ruanda, Srebrenica, Kosovo, entre otros. Ello plantea un dilema entre el respeto a los principios de soberanía y no intervención y la necesidad de proteger a las poblaciones en peligro en casos de violaciones graves, masivas y sistemáticas de sus derechos humanos y/o del derecho internacional humanitario. Cabe recordar que la prohibición del uso o la amenaza del uso de la fuerza es un fenómeno relativamente reciente, ya que la interdicción fue estableciéndose paulatinamente hasta quedar formalmente consagrada en la Carta de las Naciones Unidas, adoptada en 1945 (Márquez Carrasco 1998, 15)².

Experiencias recientes relacionadas con la crisis específicas como las de Sri Lanka y Costa de Marfil, la reciente intervención en Libia, los conflictos en curso en Siria y la República Centroafricana (RCA), entre otros, han demostrado los desafíos persistentes que surgen al momento de tener que

${ }^{2}$ C. Márquez Carrasco, Problemas actuales sobre la prohibición del recurso a la fuerza en el derecho internacional. Madrid, 1998. 
llegar a un consenso sobre el mejor modo de garantizar la aplicación oportuna y efectiva de los principios y normas que protegen los derechos humanos de las poblaciones vulnerables, generando al mismo tiempo la voluntad política común y una capacidad efectiva para prevenir o detener las atrocidades masivas.

América Latina no ha estado ausente de este debate. Por el contrario, la región ha tomado la iniciativa de llevar sus propias experiencias y perspectivas sobre este asunto, lo que brinda una lectura singular acerca del rol de la comunidad internacional y de sus responsabilidades cuando se trata de la incapacidad o la falta de voluntad de un Estado para proteger a su propia población y prevenir los crímenes en masa.

A la luz de esta difícil cuestión, el propósito de este trabajo es analizar las posturas de América Latina sobre la RdP. Sin embargo, cualquier debate sobre esta cuestión debe empezar por considerar brevemente de qué estamos hablando cuando decimos responsabilidad de proteger, a qué se refiere este principio, qué aspectos abarca y cuál es la situación actual, en relación con el contexto histórico y político que ha determinado muchas de las posturas adoptadas por los países de América Latina al momento de tener que aplicar decisiones de política exterior o fijar sus principios.

\section{2. ¿Qué es la responsabilidad de proteger?}

La utilización de medidas de intervención diplomática, económica y militar sigue siendo una de las cuestiones más polémicas en el plano de las relaciones internacionales. Como se ha señalado, no se trata de un fenómeno nuevo, dado que los tomistas ya debatían acerca de las justificaciones para llevar adelante una intervención, así como las razones para ello (Tuck, Richard 2002) $)^{3}$.

La tensión era -y sigue siendo- entre el derecho soberano de una nación a ser "libre" de la interferencia externa y el "derecho" de otros Estados a defender y proteger la dignidad humana sobre una base universal. Esa noción de soberanía que constituye la base tradicional de la política internacional atraviesa una crisis prolongada de identidad y de propósito, a lo que se añade la creciente cobertura global que realizan los medios de atrocidades masivas y la participación creciente de la sociedad civil (un fenómeno que algunos autores denominan "la empatía sin fronteras"). Todos estos factores han hecho que los debates acerca de la intervención sean aún más agudos y polémicos que en el pasado.

En su Informe sobre el Milenio, el Secretario General de las Naciones Unidas (SGONU), recordando que el Consejo de Seguridad (CSONU) no había

${ }^{3}$ R. Tuck: The Rights of War and Peace: Political Thought and the International Order from Grotius to Kant. Oxford, 2002.

Araucaria. Revista Iberoamericana de Filosofía, Política y Humanidades, año 16, n 32. Segundo semestre de 2014. Pp. 269-290. ISSN 1575-6823 e-ISSN 2340-2199 doi: 10.12795/araucaria.2014.i32.14 
actuado con decisión en Ruanda y Kosovo, planteó a los Estados Miembros la disyuntiva siguiente:

\begin{abstract}
Si la intervención humanitaria es, en realidad, un ataque inaceptable a la soberanía, ¿cómo deberíamos responder a situaciones como las de Ruanda o Srebrenica y a las violaciones graves y sistemáticas de los derechos humanos que transgreden todos los principios de nuestra humanidad común? (Secretario General de las Naciones Unidas 2000, 36-37) .
\end{abstract}

Ante este dilema que se presentaba a principios de este siglo, a partir de una iniciativa de algunos gobiernos como el canadiense y el australiano, se conformó la Comisión Internacional sobre Intervención y Soberanía de los Estados, que en 2001 emitió un Informe titulado La responsabilidad de proteger (en adelante "Informe ICISS"). Este informe procuraba encontrar una solución a la paradoja planteada por el Secretario General, para lo cual propuso fundamentalmente introducir un cambio de perspectiva al considerar la cuestión en términos de responsabilidad de proteger en lugar de hacerlo como intervención humanitaria, aunque algunos autores consideran que se trata meramente de un cambio nominal (Focarelli 2008, 194) ${ }^{5}$. En ese informe se concluía que la soberanía no solo daba al Estado el derecho de "controlar" sus asuntos, sino que le confería al Estado la "responsabilidad" primordial de proteger a la población dentro de sus fronteras. También proponía que cuando un Estado no protegiese a su población, ya fuera por falta de capacidad o de voluntad para hacerlo, la comunidad internacional podría asumir esa responsabilidad ${ }^{6}$.

El concepto de responsabilidad de proteger fue recogido posteriormente en una serie de documentos (de carácter no vinculante desde un punto de vista jurídico). Entre ellos, en su informe Un mundo más seguro: una responsabilidad compartida (en adelante "Un mundo más seguro"), el Grupo de Alto Nivel sobre las amenazas, los desafíos y el cambio, se hace eco del Informe ICISS y subraya que más que un derecho a intervenir, los Estados tienen una obligación erga omnes de tomar, cuanto antes, todas las medidas a su alcance para prevenir o poner fin a violaciones graves y masivas a los derechos humanos. En este informe se sostiene que se trata de una responsabilidad colectiva internacional, “ejercida por el Consejo de Seguridad por la que se autorizaba la intervención militar como último recurso en caso de genocidio y otras matanzas en gran escala, limpieza étnica y graves violaciones del derecho humanitario, que los gobiernos soberanos hubiesen demostrado no poder o no querer evitar". El

\footnotetext{
${ }^{4}$ Secretario General de las Naciones Unidas 2009. Informe "Hacer efectiva la responsabilidad de proteger" (Doc. A/63/677).

5 C. Focarelli, The Responsibility to Protect Doctrine and Humanitarian Intervention: Too Many Ambiguities for a Working Doctrine, "Journal of Conflict and Security Law 13" pp.191-213.

6 Para un análisis más detallado puede verse Arredondo, 2012, pp. 41 y ss.
} 
Grupo propuso ciertos criterios básicos que legitimarían la autorización del uso de la fuerza por el Consejo de Seguridad de las Naciones Unidas, incluida la gravedad de la amenaza, el hecho de que debe ser el último recurso, y la proporcionalidad de la respuesta. Como señala Focarelli, es casi un lugar común observar que las seis condiciones o principios precautorios elaborados por el ICISS y recogidos por el GAN reflejan fielmente aquellos elaborados por la tradición teológica cristiana de la guerra justa, aunque como este autor admite el problema no son las condiciones en sí mismas, sino cómo éstas son interpretadas en cada caso concreto (Focarelli 2008, 197) ${ }^{7}$.

En su informe de 2005 Un concepto más amplio de libertad: desarrollo, seguridad y derechos humanos para todos (en adelante, "Un concepto más amplio de libertad"), el SGONU sostiene la necesidad de "avanzar hacia la meta de asumir la "responsabilidad de proteger" a las víctimas posibles o reales de las atrocidades masivas y actuar en consecuencia". Para ello, propuso que, para autorizar el uso de la fuerza en general, se aplicara una lista de los criterios propuestos, que incluyesen la gravedad de la amenaza, la proporcionalidad y la posibilidad de éxito.

Por su parte, los Jefes de Estado y de Gobierno, en el Documento Final de la Cumbre Mundial de ese mismo año (en adelante, "Documento Final"), decidieron aceptar esa propuesta y parecería que han elevado la responsabilidad de proteger al rango de obligación, que, por una parte, exige a los Estados "proteger a su población del genocidio, los crímenes de guerra, la depuración étnica y los crímenes de lesa humanidad" (Naciones Unidas 2005, para. $138)^{9}$; y, por otra, en esos mismos supuestos, señala la responsabilidad de la comunidad internacional, "por medio de las Naciones Unidas", "de utilizar los medios diplomáticos, humanitarios y otros medios pacíficos apropiados, de conformidad con los Capítulos VI y VIII de la Carta, para ayudar a proteger a las poblaciones del genocidio, los crímenes de guerra, la depuración étnica y los crímenes de lesa humanidad" (Naciones Unidas 2005, para. 139) ${ }^{10}$. En el ejercicio de esa responsabilidad de "ayudar a proteger" a la población civil contra ese tipo de crímenes, los Estados se declaran dispuestos (aunque no se obligan) a llevar adelante "en cada caso concreto" una eventual acción colectiva por medio del Consejo de Seguridad, cooperando, cuando sea oportuno, con las organizaciones regionales pertinentes, "si los medios pacíficos resultan inadecuados" y hay evidencia de que las autoridades nacionales no están cumpliendo con la obligación de proteger a su población.

\footnotetext{
7 Carlo Focarelli, The Responsibility to Protect Doctrine and Humanitarian, op. cit., p.197.

${ }^{8}$ Naciones Unidas. Un concepto más amplio de la libertad: desarrollo, seguridad y derechos humanos para todos. Informe del Secretario General. Doc. A/59/2005, para. 132.

${ }^{9}$ Naciones Unidas. Documento Final. 2005. Documento Final de la Cumbre Mundial 2005. Doc. $\mathrm{A} / \mathrm{RES} / 60 / 1$.

${ }^{10}$ Naciones Unidas. Documento Final. 2005. Documento Final de la Cumbre Mundial 2005. Doc. $\mathrm{A} / \mathrm{RES} / 60 / 1$
}

Araucaria. Revista Iberoamericana de Filosofía, Política y Humanidades, año 16, n ${ }^{\circ} 32$. Segundo semestre de 2014. Pp. 269-290. ISSN 1575-6823 e-ISSN 2340-2199 doi: 10.12795/araucaria.2014.i32.14 
La comunidad internacional deberá estar dispuesta a adoptar medidas colectivas "de manera oportuna y decisiva", por medio del Consejo de Seguridad de las Naciones Unidas y de conformidad con la Carta de las Naciones Unidas, en cada caso concreto y en cooperación con las organizaciones regionales pertinentes, cuando proceda, si los medios pacíficos, que incluyen los diplomáticos, humanitarios y de otra índole, resultan inadecuados y "es evidente" que las autoridades nacionales no protegen a su población.

El principio de la RdP procura ser a la vez una respuesta tanto intelectual como práctica a la crisis de la soberanía. En ese sentido, la responsabilidad de proteger pretende subrayar la importancia de una "soberanía responsable" por parte de los Estados a quienes confía la responsabilidad primaria de proteger a su población, con el apoyo de una acción internacional concertada, y si los Estados no pueden o carecen de la voluntad de proteger a su población la comunidad internacional está habilitada para movilizar las herramientas políticas y diplomáticas, que incluyen, como último recurso, el uso de la fuerza para restaurar esa soberanía responsable.

El ejercicio de dicha responsabilidad exigía la adopción de medidas preventivas, reactivas y de reconstrucción para evitar y afrontar crisis y allanar el camino para que se instauren la justicia, el estado de derecho y el respeto de los derechos humanos. El genocidio, los crímenes de guerra, la depuración étnica y los crímenes de lesa humanidad nacen de la perpetuación en el tiempo de actos de racismo, discriminación racial, xenofobia y otras formas conexas de intolerancia. Consecuentemente, la forma de hacer efectiva la RdP requiere que una amplia variedad de actores adopten una combinación de medidas preventivas, reactivas y de apoyo. Por eso, la RdP contempla tres tipos de responsabilidades: de prevenir, de reaccionar y de reconstruir.

Asimismo, en la concreción de esas responsabilidades, el SGONU esbozó la "estructura de pilares", que comprende: la obligación de un Estado de respetar y proteger los derechos humanos de su propia población (Pilar I); sin embargo, dado que existen Estados que carecen de capacidades para cumplir con el primer pilar, la comunidad internacional tiene la obligación de brindar formación y asistencia a esa Estados (Pilar II). Sin perjuicio de ello, cuando se producen crímenes en masa en un Estado, la comunidad internacional, a través del CSONU, tiene que proveer una "respuesta oportuna y decisiva". Como el propio SGONU lo señala en su Hacer efectiva la responsabilidad de proteger (en adelante, Hacer efectiva) los tres pilares tienen la misma importancia y no hay una secuencia fija sobre qué pilar o medida debería ser usada para asegurar una respuesta adecuada a una situación de crisis (Hacer Efectiva, 10, para. 12).

El principio de responsabilidad de proteger no ha estado exento de crítica, ya que hay quienes consideran que la RdP es intervención humanitaria con un nombre diferente (Marks y Cooper 2010) ${ }^{11}$. En particular, se ha señalado que

${ }^{11}$ S. P. Marks y N. Cooper, 2010. The Responsibility to Protect: Watershed or Old Wine in a New Bottle?, "Jindal Global L.Rev". 2: 86-130

Araucaria. Revista Iberoamericana de Filosofía, Política y Humanidades, año 16, no 32. Segundo semestre de 2014. Pp. 269-290. ISSN 1575-6823 e-ISSN 2340-2199 doi: 10.12795/araucaria.2014.i32.14 
se trata de una herramienta que le da a los países poderosos una ventana de oportunidad para usar la fuerza a su propia discreción, principalmente cuando se trata de la responsabilidad de reaccionar (Chomsky 2009, Hobsbawm 2008, xiv) ${ }^{12}$. Al respecto, es preciso recordar que la $\mathrm{RdP}$ abarca tres dimensiones diferentes ya señaladas, que implican el uso de todas las herramientas disponibles de acuerdo a los Capítulos VI, VII y VIII de la Carta de la ONU, que van desde respuestas no coercitivas a la acción colectiva (Hacer Efectiva, 8, para. 10.c).

El desarrollo del principio de la RdP es un avance positivo, ya que clarifica y refuerza las obligaciones contraídas por los Estados para asegurar la protección de las poblaciones vulnerables (Asia-Pacific Centre for the Responsibility to Protect 2010). En este sentido, representa un paso importante tanto para prevenir, reaccionar y responder a actos de genocidio, crímenes de guerra, depuración étnica y crímenes contra la humanidad así como para la defensa de los principios fundamentales del derecho internacional, en particular aquellos del derecho internacional humanitario, de los refugiados y los derechos humanos. Estos principios deben ser aplicados lo más consistente y uniformemente posible, para lo cual es de crucial importancia que la alerta temprana y la evaluación se lleven a cabo de manera justa, prudente y profesional y que el uso de la fuerza siga siendo el último recurso (Parlamento Europeo 2012).

\section{América Latina ante la responsabilidad de proteger}

Antes de considerar la actual posición de los países de América Latina con relación al principio de la RdP, es importante tener en cuenta la historia de América Latina y su firme y sostenido apoyo al principio de no intervención. Por otra parte, también es relevante analizar la sólida tradición latinoamericana con respecto a la promoción, protección y defensa de los derechos humanos. En mi opinión, la combinación de estos elementos es esencial para entender las posiciones de política exterior actual de los Estados de la región vis-à-vis el principio de la RdP.

La política exterior de América Latina se fue construyendo gradualmente durante el siglo XIX y principios del siglo XX, basada en seis principios: a) la igualdad soberana de todos los Estados, b) la no intervención, c) la integridad territorial, d) la autodeterminación, e) la solución pacífica de controversias, y f) el respeto del derecho internacional. Sucesivas Conferencias Interamericanas, a partir de 1899, reforzaron estos principios, rechazando enfáticamente el

${ }^{12}$ N. Chomsky, 2009. Intervención en el Diálogo Temático sobre Responsabilidad de Proteger. AGONU, Nueva York, 23 de julio 2009; y E. Hobsbawm, 2008. Guerra y paz en el siglo XXI. Barcelona: Crítica.

Araucaria. Revista Iberoamericana de Filosofía, Política y Humanidades, año 16, n 32. Segundo semestre de 2014. Pp. 269-290. ISSN 1575-6823 e-ISSN 2340-2199 doi: 10.12795/araucaria.2014.i32.14 
intervencionismo y estableciendo prácticas humanitarias ejemplares como el asilo, a la vez que sirvieron para convencer a los Estados Unidos sobre la conveniencia de adoptar la política de "buen vecino", que condujo a una mayor cooperación y comprensión dentro del hemisferio (Petrella 2013) ${ }^{13}$.

Después de la Segunda Guerra Mundial, la adopción de la Carta de la Organización de los Estados Americanos (OEA), así como el Tratado Americano de Soluciones Pacíficas (Pacto de Bogotá), y la Declaración Americana de los Derechos y Deberes del Hombre contribuyó a consolidar estos principios en la región. En efecto, hay que recordar que la Declaración Americana de los Derechos y Deberes del Hombre precedió a la Declaración Universal de los Derechos Humanos de la ONU, y muchos de los principios del sistema interamericano fueron incorporados a la Carta de las Naciones Unidas. A partir de estos momentos históricos el papel de la OEA, con sus luces y sombras, ha servido para demostrar que los países de la región se asocian principalmente por dos razones: las necesidades estratégicas que surgen de compartir una extensa área geográfica y las afinidades culturales e institucionales, que se reflejan en valores comunes como la democracia, los derechos humanos y los principios republicanos (Lagorio 1998) ${ }^{14}$.

América Latina es una región que ha sufrido una larga historia de violaciones de los derechos humanos, intervenciones extranjeras e inestabilidad política. Las últimas décadas exhibieron un cambio significativo a través de la recuperación de la democracia, el desarrollo de instituciones y la creación de un sistema único de protección de los derechos humanos. Este sistema fomenta un mecanismo regional que contribuye a la prevención de las atrocidades en masa y sirve para reforzar la responsabilidad de los Estados en la protección de su propio pueblo. Representa un esfuerzo por superar los errores del pasado y es un ejemplo para la comunidad internacional, ya que establece mecanismos de prevención para proteger a todos los habitantes de un país.

Sin embargo, cuando se plantea la cuestión de la protección de los derechos humanos por parte de otros Estados en territorio extranjero, América Latina muestra un fuerte apoyo al principio de no intervención en los asuntos internos y una clara reticencia a apoyar cualquier tipo de intervención extranjera. Esto es principalmente debido a razones históricas, que demuestran que el intervencionismo ha sido utilizado como un instrumento de la política exterior en América Latina, principalmente por los Estados Unidos; pero también hay muchos otros ejemplos de intervenciones extranjeras por parte de las potencias hegemónicas de cada momento histórico (Podestá Costa 1961, 972-973, Caminos 1996, 18). Los países de América Latina continúan considerando el principio de no intervención como la piedra basal sobre la que se asienta su

${ }^{13}$ F. Petrella, 2013. John Kerry, la OEA y la Argentina, Infobae, 18 de diciembre de 2013.

${ }_{14}$ R. Lagorio, 1998. Institutionalization, Cooperative Security, and Peacekeeping Operations. En Dominguez, Jorge, Latin America and the Caribbean in the Post-Cold War Era. University of Pittsburgh Press, Pittsburgh.

Araucaria. Revista Iberoamericana de Filosofía, Política y Humanidades, año 16, $\mathrm{n}^{\circ}$ 32. Segundo semestre de 2014 Pp. 269-290. ISSN 1575-6823 e-ISSN 2340-2199 doi: 10.12795/araucaria.2014.i32.14 
marco de relaciones internacionales y por ello sostienen que "la doctrina de la intervención humanitaria es incompatible con las Cartas de las Naciones Unidas y de la OEA" (Caminos 1996, 20) ${ }^{15}$.

Un factor adicional a considerar es que, si bien muchos observadores están acostumbrados a pensar América Latina como un sujeto monolítico, la región muestra una saludable diversidad política e ideológica que se ve reflejada en las posiciones de política exterior que adoptan sus Gobiernos y que no necesariamente concuerda con posturas sostenidas por esos mismos países en el pasado.

Esa heterogeneidad incide en ciertas alianzas regionales en la medida en que las tensiones geopolíticas del hemisferio se traducen en "fronteras ideológicas" frente a los asuntos que ocupan la agenda de política exterior de nuestros países. En el caso de la responsabilidad de proteger, a grandes rasgos, podrían esbozarse tres tipos de fronteras ideológicas: dos con una posición clara y definida: la bolivariana y la interamericana; y una tercera, que podríamos denominar ecléctica, en la medida en que toma elementos de las dos primeras, para situarse en una posición intermedia entre ambas.

La frontera bolivariana, en la que podríamos ubicar a Cuba, Venezuela, Nicaragua, Bolivia y Ecuador, parte de una concepción "anti-imperialista" que ubica a Latinoamérica en una posición contrapuesta a la potencia hemisférica, los Estados Unidos. Por ende, busca reforzar el principio no intervención, rechazando cualquier forma de injerencia externa. Desde esta óptica, se percibe a los Estados Unidos como "el enemigo" de la soberanía nacional, un valor primordial de esta postura, y se supone a la RdP como un instrumento encubierto del hegemón que busca intervenir en la región para proteger o imponer sus propios intereses.

La frontera interamericana es la que congrega a la mayoría de los Estados de la región, en donde se agrupan tanto gobiernos de izquierda (Uruguay, El Salvador), como de derecha o centro (México, Chile, Colombia, Perú), países que sin dejar de defender el principio de no intervención consideran que la responsabilidad de proteger representa un elemento positivo en la protección de los derechos humanos en casos de crisis humanitarias y, por lo tanto, propician la afirmación del principio.

Finalmente, la frontera ecléctica es aquella que agrupa a Estados como Brasil y Argentina, que si bien mantienen una posición de firme aserción en lo que se refiere a la promoción y protección de los derechos humanos, esa postura no se traduce en una clara aceptación del principio de la responsabilidad de proteger. A pesar de que parecen haber acogido favorablemente el principio de la responsabilidad de proteger, estiman que dicho principio, sobre el que albergan

\footnotetext{
${ }^{15}$ H. Caminos, 1996. La Intervención Humanitaria ante el Derecho Internacional. Anticipo de 34 “Anales". Academia Nacional de Derecho y Ciencias Sociales de Buenos Aires.
} 
algunas dudas, necesita una mayor elaboración, particularmente en cuanto a la responsabilidad de reaccionar y el eventual uso de la fuerza. En el caso de Argentina, su apoyo a los principios de soberanía nacional y de no injerencia en los asuntos internos es persistente en su discurso, pero Argentina también se considera un "Campeón" de la RdP. Por su parte, Brasil, con un enfoque más cauteloso, parecería haber dado un paso adicional cuando propuso el concepto de responsabilidad al proteger ( $\mathrm{RaP}$ ) en el debate de la ONU de 2011, aunque este concepto parece ser tanto criticar y a la vez validar el principio de la RdP.

Este mapa plural de la política latinoamericana genera dificultades al momento de abordar la cuestión en los organismos o grupos regionales, ya que, con frecuencia, las agendas geopolíticas basadas en alianzas ideológicas obstruyen la posibilidad de alcanzar un consenso en la materia. En ese sentido, las fronteras ideológicas, especialmente las promovidas por el ALBA, pueden convertirse en un serio obstáculo en la medida que los otros gobiernos de la región no sepan defender la perspectiva interamericana que la mayoría comparte. A título de ejemplo, pueden mencionarse los graves sucesos ocurridos en Venezuela de 2014 durante los meses de enero y febrero de 2014, que no permiten albergar esperanzas de una rápida reacción de los países de la región, ya la Administración de Maduro, como sucedió antes con el régimen de Chávez, afirma respetar y acatar los principios democráticos y los derechos humanos, mientras que la realidad parece ser la opuesta (OEA 2014).

La primera reacción de los países latinoamericanos al informe "Hacer efectiva" fue de cierta cautela. Muchos países comparten la premisa que la inclusión del concepto de la responsabilidad de proteger en el Documento Final de la Cumbre Mundial de 2005 fue un logro, pero consideran que la redacción acordada contiene vulnerabilidades que hacen que sea necesario avanzar a paso lento en la implementación del concepto. Algunos países consideran positivo que el tema sea abordado en el seno de la AGONU y coinciden en que sería deseable que ese debate desemboque en el dictado de una resolución. Sin embargo, luego de cinco años y sendos informes del SGONU, la Asamblea General no ha incluido el tema formalmente en su agenda. Esta situación hizo que el Representante de Guatemala, país que presentó el proyecto de la primera resolución de la Asamblea General sobre la RdP (Res. 63/308), instara al organismo a incluir esta cuestión como "un tema de la agenda formal para aclarar el camino a seguir" ${ }^{\prime 16}$.

Con relación a la estructura de pilares, en general los países latinoamericanos consideran que no existen controversias respecto al primer pilar, que establece que cada Estado tiene la responsabilidad de proteger a su propia población.

${ }^{16}$ Intervención realizada en nombre de la Misión Permanente de Guatemala ante las Naciones Unidas. Asamblea General Diálogo Temático Interactivo sobre "La responsabilidad de proteger: la responsabilidad del Estado y la Prevención", 11 de septiembre 2013.

Araucaria. Revista Iberoamericana de Filosofía, Política y Humanidades, año 16, $\mathrm{n}^{\circ} 32$. Segundo semestre de 2014 Pp. 269-290. ISSN 1575-6823 e-ISSN 2340-2199 doi: 10.12795/araucaria.2014.i32.14 
Lo mismo puede decirse del segundo (formación de capacidad y asistencia), aunque hay algunos Estados que hacen referencia a la conveniencia de debatir y elaborar más sobre el tema. El tercer pilar, referido a la "respuesta oportuna y decisiva", es claramente el más controvertido ya que abre la posibilidad de aplicar el Capítulo VII de la Carta. En ese sentido, debe señalarse que persiste cierta sensibilidad ante los eventuales riesgos de abusos a que pudiera dar lugar la aplicación del concepto en la práctica, conjeturas que en cierto modo probaron tener sustento con hechos ocurridos con motivo de las acciones de la OTAN en Libia en 2011.

Desde los inicios del debate sobre la RdP en América Latina pudo percibirse una clara divisoria de aguas entre algunos países que deseaban impulsar el desarrollo del principio, de acuerdo la estrategia esbozada por el Secretario General en su primer informe, encabezados por México y Chile; mientras que en el extremo opuesto se situaron algunos países con una visión radicalmente distinta del principio, que se preocuparon por enfatizar en los riesgos de la adopción de una norma que contenga el principio de la RdP. No hubo desacuerdo en torno a los dos primeros pilares, ya que existe consenso en sostener que la responsabilidad de proteger a la propia población es uno de los roles principales del Estado.

Si bien hubo algunos países y ONGs que intentaron relacionar la RdP con la agenda del desarrollo (argumentando que los supuestos donde se aplicaría la responsabilidad de proteger se dan en países menos desarrollados, lo que haría ineludible atacar las causas de esa pobreza) ${ }^{17}$, la mayoría de los Estados se opuso a esa vinculación. Sin desconocer el vínculo existente entre el desarrollo, derechos humanos y seguridad (Secretario General ONU 2005), se estimó que debatir la RdP en el contexto de las causas de los conflictos produciría una dispersión del debate, cuando el objetivo perseguido es precisamente el opuesto. Lo que se pretende en el contexto actual es hacer operativo lo que los Jefes de Estado acordaron en 2005, es decir, transformar un principio de naturaleza política en normas jurídicas, para lo cual es menester concentrar el debate para arribar a resultados concretos en el menor tiempo posible.

Sin dudas, el tercer pilar continúa siendo el más controvertido por las razones expuestas precedentemente. Si el debate sobre la RdP se centrara sólo en este aspecto, se corre el riesgo de que no poder llevar adelante una discusión amplia y comprensiva de los otros dos pilares, con el peligro consiguiente de una paralización del diálogo y las negociaciones. En este punto, algunos países han sostenido que no resulta posible avanzar en la operacionalización del concepto

${ }^{17}$ Cabe recordar que en mayo de 2005, antes de que el concepto fuera formalmente adoptado en la Cumbre de Naciones Unidas, Chile, México y Japón habían elaborado un non paper en el que sostenían que la noción, en su faceta preventiva, debía dirigirse a las raíces del conflicto, como la incitación al odio entre grupos étnicos y la desigualdad al interior de cada país, abarcando el desarrollo y la construcción de capacidades (Muñoz, 2010, 104).

Araucaria. Revista Iberoamericana de Filosofía, Política y Humanidades, año 16, n ${ }^{\circ} 32$. Segundo semestre de 2014. Pp. 269-290. ISSN 1575-6823 e-ISSN 2340-2199 doi: 10.12795/araucaria.2014.i32.14 
sin una reforma del Consejo de Seguridad, que incluya una limitación o, eventualmente, la eliminación de la utilización del derecho de veto de los cinco miembros permanentes en esta materia. Sin embargo, otros países consideran que la discusión sobre la reforma del Consejo es mucho más amplia y ya lleva más de veinte años sin que se hayan producido avances sustanciales al respecto. Por ello, subordinar el debate sobre la RdP a esta cuestión implicaría posponer sine die la implementación del concepto. Por otra parte, en situaciones donde se están produciendo violaciones masivas a los derechos humanos y/o al derecho internacional humanitario resultaría difícil argumentar que la inacción del Consejo se encuentra supeditada a una eventual y futura reforma del Consejo. Detrás de esta postura, se enrolaron algunos países que consideran que hay que avanzar de manera pragmática con las actuales reglas de juego.

Un tema que fue particularmente objeto de debate fue la afirmación del Secretario General de que, en caso de parálisis del CSONU, la Asamblea General podría adoptar medidas coercitivas, en el marco de lo establecido por la Resolución 377 (V) "Unión pro Paz". Ello generó controversia en la medida en que, si bien se trataría de una situación excepcional, se aparta de lo dispuesto textualmente en los párrafos 138 y 139 del Documento Final y de lo establecido en la propia Carta. En mi opinión, si bien esta posibilidad no está expresamente contemplada en los instrumentos mencionados, no estaría mal utilizarla en la medida en que la acción coercitiva que eventualmente se adopte gozaría de mayor consenso y, por lo tanto, tendría mayor legitimidad. Si hay consenso en cuanto a que, en caso que se decidiera su aplicación, las acciones coercitivas son un último recurso y deberían adoptarse caso por caso, evitando todo tipo de automaticidad.

Las posiciones generales fueron reiteradas por los Estados latinoamericanos en los cinco Diálogos temáticos sobre la Responsabilidad de Proteger que se llevaron a cabo anualmente en la AGONU entre 2009 y 2013. Cada uno de estos Diálogos Temáticos fue precedido por un informe del SGONU que ayudó a centrar el debate sobre un determinado aspecto del principio. Una mirada cercana a las declaraciones de los representantes de los países de América Latina evidencia de que hubo escasas modificaciones respecto de las diferentes posiciones adoptadas por los Estados de la región. De estas y otras notas de prensa, documentos y posiciones adoptadas en los diferentes foros, es posible extraer tres diversas posturas sobre RdP:

\section{Los partidarios de la responsabilidad de proteger}

Chile y México aparecen como los "campeones" en la promoción del principio, al señalar incluso que éste habría adquirido el rango de norma jurídica. Chile, refiriéndose a la naturaleza jurídica de la responsabilidad de 
proteger, sostuvo que el principio, al ser adoptado en la Cumbre de 2005, había adquirido el carácter de norma ${ }^{18}$ y expresó su "decidido compromiso con la RdP, cuyas bases sólidas quedaron establecidas por los Jefes de Estado y de Gobierno en los párrafos 138 y 139 del Documento Final, y que no pueden ser selectivamente abordados ni revisados". En cuanto al uso de la fuerza, manifestó que el imperativo colectivo no es intervenir, sino adoptar cualquier acción "oportuna y decisiva", de conformidad con la Carta de la ONU, ya que en el Documento Final "no hay automaticidad, ni gatillos, ni luces verdes implícitas para el uso de la fuerza" (Chile 2009). México considera que debería desarrollarse una práctica gradual que fortalezca la acción multilateral y consolide el rol de las Naciones Unidas "para responder a situaciones similares a las que, en el pasado, sólo ha observado de perfil o, peor aún, ha tenido que convalidar ex post facto" (México 2009).

Otros países de la región, como Costa Rica, Guatemala, Colombia, Uruguay, Panamá ${ }^{19}$, entre otros, también han expresado su continuado apoyo al compromiso adoptado en 2005, enfatizando que cualquier intento de extender el concepto a otros casos, o de asociarlo con otras nociones, queda fuera del acuerdo alcanzado en el Documento Final.

Uruguay reiteró su apoyo al consenso alcanzado en 2005 y recordó que:

The prohibition to commit genocide, ethnic cleansing, crimes against humanity and war crimes are not only obligations within the framework of the Human Rights International Law, but are real jus cogens norms of absolute respect and inviolability (Uruguay 2012).

Por otra parte, teniendo en cuenta la lección de la intervención en Libia, Uruguay subrayó la necesidad de separar la RdP de nociones imprecisas que no tienen nada que ver con el consenso de 2005, por ejemplo la utilización del uso de la fuerza para otros fines que no sean prevenir o detener las atrocidades masivas, como “cambio de régimen” (Uruguay 2013).

\section{Los adversarios de la RdP}

En una posición opuesta se ubican países como Venezuela, Nicaragua, Bolivia, Cuba y Ecuador, que expresan un fuerte rechazo a la RdP, sosteniendo

\footnotetext{
${ }^{18}$ Citando palabras del jurista británico Ian Brownlie, adujo que "El Acta Final u otra declaración de conclusiones de una conferencia entre Estados puede ser una forma de tratado multilateral" y, en todo caso, es posible considerarlas como una fuente del derecho internacional. Más aun, la práctica de órganos políticos como la AG, cuyas resoluciones no son vinculantes, tiene según Brownlie "considerable significado legal".

${ }^{19}$ Se puede acceder a la mayoría de los discursos de los diferentes países de la región en el sitio de la Coalición Internacional para la RdP en http://www.responsibilitytoprotect.org.
} 
que resulta "debatible" definir cuál es la mejor forma de responder a las crisis humanitarias de manera previsible, sostenible y efectiva, sin condiciones previas ni dobles raseros. Por ejemplo, Nicaragua reivindica los principios de soberanía y no intervención y recuerda cómo

El legado del colonialismo da a los países en desarrollo razones fundadas para temer que motivos dignos de elogio puedan acabar siendo manipulados, una vez más, para justificar intervenciones arbitrarias y selectivas contra los Estados más débiles (Nicaragua 2009).

Asimismo, procura desviar el foco del debate, afirmando que el modo de resolver las crisis es atacando "las causas de fondo", que son "el subdesarrollo y la exclusión social". Sostiene que el principio de la RdP no se aplica equitativamente, sino selectivamente, con lo cual se "socava el derecho internacional". Asimismo, afirma que la RdP no es necesaria y, a la inversa, tampoco garantiza que los Estados intervendrán para prevenir otra Ruanda. Por ello, D’Escoto finalizó abogando por la aplicación eficaz del sistema de seguridad colectiva vigente.

De modo similar, Venezuela sostiene que en este debate "no existen normas vinculantes" y alertó contra "el predominio que tienen en el mundo de hoy... las potencias imperiales dominantes, cuyos intereses determinan generalmente el rumbo de la dinámica de las relaciones internacionales". En una curiosa interpretación del esquema de seguridad vigente en la Carta de las Naciones Unidas, Venezuela considera que los elementos de la propuesta del Secretario General requerirán, necesariamente, de una reforma sustantiva de la Carta de las Naciones Unidas (Venezuela 2009). Sin embargo, Venezuela apoya el establecimiento de un proceso intergubernamental dentro de la Asamblea General para que este tema sea discutido formalmente (Venezuela 2013).

\section{Los eclécticos}

La frontera ecléctica reúne a Estados, como Brasil y Argentina, que si bien mantienen una posición de vigoroso apoyo a la promoción y protección de los derechos humanos, no parecen apoyar plenamente el principio de la RdP. Retóricamente apoyan la responsabilidad de proteger, pero también se han expresado algunas dudas al respecto. Brasil y Argentina sostienen que la RdP necesita una mayor elaboración, sobre todo en los aspectos referidos a la responsabilidad de reaccionar y el eventual uso de la fuerza. En el caso de Argentina, el apoyo a los principios de soberanía nacional y de no intervención es persistente en su discurso; sin embargo Argentina también es tenida por un "Campeón” de la RdP. 
Brasil, considerando que todos los Estados Miembros de las Naciones Unidas están a favor de la promoción y protección de los derechos humanos, instó a evitar visiones maniqueas entre quienes supuestamente serían defensores de los derechos humanos y aquellos que no. Se pronunció a favor de no reabrir el concepto, así como señaló que tampoco debería aplicárselo a supuestos que están más allá de los cuatro crímenes enumerados, como el sida, los desastres naturales o el cambio climático. Brasil fue claro sobre la naturaleza jurídica del principio, señalando que nunca ha sostenido que la $\mathrm{RdP}$ sea propiamente un principio, y mucho menos una nueva norma jurídica, sino que se trata de un fuerte llamado político a todos los Estados para que se atengan a las obligaciones previamente asumidas en la Carta, en importantes convenciones de derechos humanos, en el derecho internacional humanitario y en otros instrumentos (Brasil 2009).

Ello no obstante, Brasil, reafirmando el principio de "no indiferencia", señaló que existe un marco legal para actuar en casos de crisis humanitarias y que la falta de implementación del concepto no puede ser alegada como excusa para dejar que estos crímenes se lleven a cabo. Refiriéndose a la estructura de pilares, se manifestó a favor de desarrollar los pilares I y II aunque, en contra de lo sostenido por el Secretario General en su Informe, afirmó que el tercer pilar es subsidiario del primero y que se trata de un curso de acción de naturaleza excepcional (Brasil 2009).

Este punto de vista fue mantenido por Brasil dos años más tarde, cuando presentó su documento de concepto sobre la responsabilidad al proteger. Mientras formó parte como Miembro no permanente del CSONU Brasil votó a favor de las Resoluciones 1970 y 1973, que permitieron el uso de la fuerza en el caso de Libia, y posteriormente elaboraron una nueva doctrina complementaria de la RdP, llamada "responsabilidad al proteger" (Brasil 2011). Esta doctrina sostiene principalmente que la comunidad internacional debe mostrar una gran responsabilidad en el ejercicio de su responsabilidad de proteger. Brasil apoya una visión restrictiva en cuanto al uso de la fuerza, afirmando que sólo puede ser utilizado como último recurso y siempre con la autorización del Consejo de Seguridad, de conformidad con el Capítulo VII de la Carta, o, en circunstancias excepcionales, por la Asamblea General, de conformidad con su Resolución 377 (V).

Desde un punto de vista jurídico, la RaP sostenida por el Gobierno de Brasil subrayó la necesidad de establecer reglas claras para definir la forma en que una intervención debería ser aprobada e implementada (Rodrigues 2012). Aunque esta iniciativa fue presentada por la Presidente de Brasil, Dilma Roussef, este país no le ha adecuado seguimiento y ha sido criticado fuertemente por su acción casi unilateral en la presentación de la RaP. 
La Argentina ha manifestado su voluntad de seguir contribuyendo a la búsqueda de respuestas a crisis humanitarias como las que la comunidad internacional ha presenciado en los tres últimos lustros. En ese sentido, manifestó su clara preferencia hacia la utilización de los mecanismos de seguridad colectiva previstos en la Carta de las Naciones Unidas y su renuencia a aceptar que la RdP sea ejercida de modo unilateral por un Estado, una coalición de Estados o una organismo regional sin contar con una autorización expresa del CSONU. Asimismo, la República Argentina ha puesto de manifiesto que el Consejo ya cuenta con el marco jurídico y las herramientas para poner en marcha esa responsabilidad de reaccionar frente a violaciones graves, masivas y sistemáticas de los derechos humanos y del derecho humanitario. Por lo tanto, lo que se requiere es la voluntad de sus miembros y, en particular, de sus miembros permanentes (P5) para poder ejercer de modo eficaz y legítimo esa responsabilidad subsidiaria que recae sobre la comunidad internacional en su conjunto.

La Argentina mostró un claro respaldo a la noción de responsabilidad de proteger, al afirmar que:

La responsabilidad de proteger es una obligación esencial de los Estados, puesto que no hace más que conjugar todas sus obligaciones internacionales de protección de la persona humana. No obstante, cuando éstos fallan, las Naciones Unidas no pueden mantenerse ajenas y su acción puede evitar la comisión de estos graves crímenes. La implementación de la Responsabilidad de Proteger requiere un debate cuidadoso y detallado y la Asamblea General es el espacio adecuado para dicho proceso (Argentina 2010).

Cuando se suscitó la situación en Libia a principios de 2011, la Argentina expresó "su profunda preocupación por la grave situación", al tiempo que lamentaba "la pérdida de vidas y los actos de violencia acaecidos en los enfrentamientos". Asimismo, instó a "una pronta solución pacífica, dentro de un diálogo democrático constructivo y de absoluto respeto de los derechos humanos y de la voluntad del pueblo libio" (Argentina 2011a). Del mismo modo, el Gobierno argentino expresó su profunda preocupación por las graves violaciones a los derechos humanos en Libia, y ante el llamamiento de la Alta Comisionada para los Derechos Humanos copatrocinó la convocatoria de una sesión especial del Consejo de Derechos Humanos, que requirió el cese inmediato de las graves violaciones a los derechos humanos cometidas por las autoridades de Libia, y lanzó una investigación internacional independiente sobre la violenta represión a las manifestaciones en ese país (Argentina 2011b). Sin embargo, la Argentina rechazó expresamente la inclusión de medidas propuestas de conformidad con el Capítulo VII de la Carta de la ONU, lo que condicionó a una constatación de la situación en el terreno a través de datos objetivos, serios y concluyentes. 
No hace falta decir que este condicionamiento tenía como finalidad a prevenir el tipo de acción que posteriormente se desencadenó debido a la autorización concedida por Resoluciones 1970 y 1973 del CSONU, sobre las que pesan fuertes críticas. Estas Resoluciones representan la primera instancia en que un caso de responsabilidad de proteger condujo a una intervención armada. La adopción de la Resolución 1973 contó con el apoyo de la Liga Árabe y la Unión Africana, lo que le confirió una legitimidad incuestionable al principio (Garrigues 2011) ${ }^{20}$, más allá de la abstención de China y Rusia. La resolución estableció una zona de exclusión aérea y un embargo naval en Libia, y autorizó a los Estados miembros a adoptar todas las medidas necesarias para proteger a los civiles, aunque excluyó expresamente la utilización de una fuerza de ocupación extranjera de cualquier tipo en cualquier parte del territorio libio (Res. 1973 /2011). Estas condiciones fueron violadas por la OTAN, en una acción que se transformó en una operación de cambio de régimen y que terminó socavando el principio de la responsabilidad de proteger.

\section{Conclusiones}

La diversidad política e ideológica en America Latina no permite avizorar un consenso sobre el principio de responsabilidad de proteger en la región, sino tres tendencias principales entre los países de la región en su aproximación a este principio. Por un lado, hay un grupo de países que promueven una fuerte oposición en contra del principio. Este grupo está representado por Venezuela, Cuba y otros países del ALBA, cuya crítica a la RdP se basa en la prevalencia de los principios de soberanía estatal y no intervención. Un segundo grupo de países se caracteriza por un apoyo constante a la responsabilidad de proteger, como es el caso de México, Panamá, Chile, Uruguay, Costa Rica y Guatemala, entre otros. Un tercer grupo, con países como Argentina y Brasil, con un discurso muy enérgico a favor de los derechos humanos, una larga historia de apoyo a las misiones de la ONU y un papel activo en el desarrollo del principio de la $\mathrm{RdP}$, a pesar de sus diferentes perspectivas. En el caso de Argentina, su apoyo a los principios de respeto de la soberanía estatal y no intervención es persistente en su discurso, pero Argentina también es considerado como un defensor de la RdP, teniendo en cuenta que su papel activo en la promoción del principio desde sus inicios. Por otra parte, Brasil, con un enfoque más cauteloso, ha dado un paso adicional cuando presentó el concepto de responsabilidad al proteger en el debate de la ONU en 2011, que tanto crítica como valida el principio de la RdP. Sin embargo, Brasil ha carecido de acción en el seguimiento de

\footnotetext{
${ }^{20}$ J. Garrigues 2011: Libia: del precedente positivo a la frustración colectiva. "Notes Internacionals 37", CIDOB. Barcelona.
}

Araucaria. Revista Iberoamericana de Filosofía, Política y Humanidades, año 16, n ${ }^{\circ} 32$. Segundo semestre de 2014. Pp. 269-290. ISSN 1575-6823 e-ISSN 2340-2199 doi: 10.12795/araucaria.2014.i32.14 
esta iniciativa y ha criticado fuertemente por su acción casi unilateral en la presentación de la RaP.

En este contexto, dos tradiciones jurídicas luchan por imponer sus propios principios a la política exterior de los gobiernos de América Latina: por un lado, como se mencionó, la tradición de respeto irrestricto a la soberanía del Estado y la defensa del principio de no intervención; y, por otro, una fuerte tradición de promoción y protección de los derechos humanos, particularmente relevante después de la desaparición de las dictaduras militares en los años ochenta.

Un análisis de las posiciones de los Estados de América Latina muestra que, si bien un gran número de gobiernos ha aceptado el principio de responsabilidad de proteger, muchos de ellos todavía están renuentes a legalizar el uso unilateral de la fuerza armada bajo pretextos humanitarios, especialmente a la luz de los abusos que han ocurrido en el pasado. En este sentido, la mayoría de los Estados no se sienten inclinados a abrir la norma del Artículo 2.4 de la Carta de las Naciones Unidas con el fin de incluir una excepción para la intervención humanitaria (Schachter 1984, 1692; Goodman 2006, 109) . $^{21}$.

Las mesas redondas regionales convocadas por la ICISS, así como las posteriores consultas sobre el informe con las ONGs y los gobiernos, coordinados por Canadá y otras organizaciones de la sociedad civil, sirvieron para evidenciar que, en la mayoría de los casos, había una hostilidad generalizada hacia el concepto de la intervención humanitaria y un amplio consenso en contra de la idea de un supuesto -derecho de injerencia-, sobre todo cuando ese supuesto derecho estaba asociado con acciones unilaterales de los Estados (World Federalist Movement 2003, Morada 2006) ${ }^{22}$.

Pero este profundo escepticismo hacia la intervención no necesariamente se traduce en un rechazo a la finalidad subyacente de la RdP: la prevención de genocidios y atrocidades masivas y la protección de las poblaciones vulnerables. La adopción de un lenguaje centrado en los derechos de las poblaciones y no en un supuesto derecho de intervención contribuyó a que una amplia gama de actores estatales y de la sociedad civil expresara su disposición a reconocer que la soberanía conlleva responsabilidades y que una intervención internacional puede ser legítima en ciertas circunstancias.

La intervención militar en Libia de 2011 puso en evidencia la necesidad de clarificar el papel de las organizaciones regionales y subregionales en la aplicación de la RdP. Si bien estas organizaciones pueden ser tanto legitimadoras como agentes operativos en la implementación de la RdP, a menudo carecen

${ }^{21}$ O. Schachter, 1984: The Right of States to Use Armed Force, "Michigan Law Review 82"; y R. Goodman, 2006: Humanitarian Intervention and Pretext for War, "American Journal of International Law", 100 (1) (2006).

22 World Federalist Movement and International Policy Group. 2003. "Civil society perspectives on the responsibility to protect, Final Report". 30 de abril de 2003; y N. Morada, 2006. R2P Roadmap in Southeast Asia: Challenges and Prospects, "UNISCI” Discussion Papers 11. 
de las capacidades y los recursos para llevar a cabo dichas operaciones de una manera significativa.

Las tres tendencias y las dos tradiciones mencionadas plantean una serie de preguntas sobre la validez del principio de la $\mathrm{RdP}$ en la región. En primer lugar, cuál seria la utilidad de la aplicación del principio en América Latina, teniendo en cuenta la existencia de un sistema preventivo, tal como el establecido en el sistema interamericano de derechos humanos. En segundo lugar, cómo articular las diferentes posiciones de política exterior de los países de la región, en particular en el contexto de un proceso muy dinámico del regionalismo y la transformación regional y la fuerte prevalencia de los principios de soberanía nacional y la no intervención. En tercer lugar, cómo impacta la participación de varios países de América Latina en operaciones de paz y cuál es la influencia de esa experiencia en America Latina y en el debate sobre la cuestión de la intervención humanitaria. Por último, cual es el papel que juegan las organizaciones de la sociedad civil y las redes en la prevención en una región donde prevalecen los sistemas democráticos.

La mayoría de los Estados de América Latina han expresado su voluntad de seguir contribuyendo a la búsqueda de respuestas a las crisis humanitarias, su clara preferencia por la utilización de los mecanismos de seguridad colectiva previstos en la Carta de las Naciones Unidas y, consecuentemente, su renuencia a aceptar que la RdP sea ejercida unilateralmente por un Estado, una coalición de Estados o un organismo regional sin la autorización expresa del Consejo de Seguridad. Asimismo, la mayoría de los países latinoamericanos ha manifestado que el Consejo de Seguridad ya cuenta con el marco jurídico y los instrumentos necesarios para poner en práctica esa responsabilidad de reaccionar a violaciones graves, masivas y sistemáticas de los derechos humanos y del derecho internacional humanitario. Lo que se requiere es la voluntad de sus Estados Miembros del CSONU y, en particular, del P5 para el ejercicio de una manera eficaz y legítima de esa responsabilidad subsidiaria impuesta a la comunidad internacional en su conjunto.

Una porción importante de América Latina está cambiando y está dispuesta a seguir apoyando la RdP, siempre y cuando el sentido común y el derecho internacional prevalezcan y no se vean arrastrados al juego unilateral de los países mas poderosos del sistema. 


\section{Referencias bibliográficas:}

Argentina. 2010. Intervención de la delegación argentina en el Diálogo Temático de la Asamblea General sobre Responsabilidad de Proteger, 9 de agosto de 2010.

Argentina. 2011. Ministerio de Relaciones Exteriores y Culto. "Situación en Libia: Argentina copatrocina la convocatoria a una sesión especial del Consejo de Derechos Humanos". Información de prensa Nº 067/11, 23 de febrero de 2011.

Argentina. 2011a. Ministerio de Relaciones Exteriores y Culto. "Situación en Libia: profunda preocupación del gobierno argentino". Información de prensa $\mathrm{N}^{\circ}$ 064/11, 22 de febrero de 2011.

Arredondo, Ricardo. 2012. Intervención humanitaria y responsabilidad de proteger ¿Hacia un nuevo paradigma de protección de los derechos humanos? Buenos Aires: Catálogos.

Arredondo, Ricardo (Director).2012. Manual de Derecho Internacional Público. La Ley - Thomson Reuters.

Arredondo, Ricardo. 2012. "La República Argentina y la responsabilidad de proteger. ¿Un atisbo de cambio?”. Pensamiento Propio (35), Coordinadora Regional de Investigaciones Económicas y Sociales, Buenos Aires.

Brasil. 2011. Carta Representante Permanente de Brasil ante las Naciones Unidas al Secretario General, fechada 9 de noviembre de 2011. Doc. A/66/551-S/2011/701.

Brasil. 2009. Intervención de la Representante Permanente de Brasil ante las Naciones Unidas, María Luiza Ribeiro Viotti, en el Diálogo Temático de la Asamblea General sobre Responsabilidad de Proteger. Nueva York, 23 de julio de 2009.

Caminos, Hugo. 1996. "La Intervención Humanitaria ante el Derecho Internacional". Anticipo de 34 Anales. Academia Nacional de Derecho y Ciencias Sociales de Buenos Aires.

Comisión Internacional sobre Intervención y Soberanía de los Estados. 2001. La responsabilidad de proteger. Doc. A/57/303.

Chile. 2009. Intervención del Representante Permanente de Chile ante las Naciones Unidas, Heraldo Muñoz, en el Diálogo Temático de la Asamblea General sobre Responsabilidad de Proteger. Nueva York, 23 de julio de 2009.

Chomsky, Noam. 2009. Intervención en el Diálogo Temático sobre Responsabilidad de Proteger. AGONU, Nueva York, 23 de julio 2009.

Focarelli, Carlo. 2008. "The Responsibility to Protect Doctrine and Humanitarian Intervention: Too Many Ambiguities for a Working Doctrine". Journal of Conflict and Security Law 13 (2):191-213. 
Garrigues, Juan. 2011. "Libia: del precedente positivo a la frustración colectiva". Notes Internacionals 37, CIDOB. Barcelona.

Goodman, Ryan. 2006. "Humanitarian Intervention and Pretext for War". American Journal of International Law, 100 (1) (2006).

Hobsbawm, Eric. 2008. Guerra y paz en el siglo XXI. Barcelona: Crítica.

Lagorio, Ricardo. 1998. "Institutionalization, Cooperative Security, and

Peacekeeping Operations". En Dominguez, Jorge Latin America and the Caribbean in the Post-Cold War Era. University of Pittsburgh Press, Pittsburgh.

Marks, Stephen P. y Cooper, Nicholas. 2010. "The Responsibility to Protect: Watershed or Old Wine in a New Bottle?" Jindal Global L. Rev. 2: 86-130. Márquez Carrasco, María del Carmen. 1998. Problemas actuales sobre la prohibición del recurso a la fuerza en derecho internacional. Madrid: Tecnos.

Mexico. 2009. Intervención del Representante Permanente de México ante las Naciones Unidas, Socorro Rovirosa, en el Diálogo Temático de la Asamblea General sobre Responsabilidad de Proteger. Nueva York, 24 de julio de 2009.

Morada, Noel M. 2006. "R2P Roadmap in Southeast Asia: Challenges and Prospects”, UNISCI Discussion Papers 11.

Muñoz, Heraldo. 2010. "La responsabilidad de proteger: tres pilares y cuatro crímenes". Foreign Affairs Latinoamérica 10.

Naciones Unidas. Documento Final. 2005. Documento Final de la Cumbre Mundial 2005. Doc. A/RES/60/1.

Naciones Unidas. Informe del Grupo de Alto Nivel sobre las amenazas, los desafios y el cambio: "Un mundo más seguro: una responsabilidad compartida”. 2004. Documento A/59/565.

Nicaragua. 2009. Intervención del Representante Permanente de Nicaragua ante las Naciones Unidas y Presidente de la $63^{\mathrm{a}}$ AGONU, Miguel D’Escoto Brockmann, en el Diálogo Temático de la Asamblea General sobre Responsabilidad de Proteger. Nueva York, 23 de julio de 2009.

OEA. 2014. Comunicado de Prensa. "Secretario General de la OEA apela a la responsabilidad de los actores políticos en Venezuela para evitar más violencia". 17 de febrero de 2014.

Parlamento Europeo. 2012. Recommendation to the Council of 18 April 2013 on the UN principle of the 'Responsibility to Protect' ('R2P') (2012/2143(INI)).

Petrella, Fernando. 2013. “John Kerry, la OEA y la Argentina”, Infobae, 18 de diciembre de 2013.

Podestá Costa, Luis Alberto. 1961. Derecho Internacional Público. Vol. I. Buenos Aires: Editorial TEA. 
Rodrigues, Gilberto M. A. 2012. "Statement". En Civil Society Perspectives on Responsibility While Protecting. International Coalition for the Responsibility to Protect, disponible en http://responsibilitytoprotectblog. files.wordpress.com/2012/09/responses_final.pdf (accedido 28-2-14).

Schachter, Oscar. 1984. "The Right of States to Use Armed Force". Michigan Law Review 82.

Secretario General de las Naciones Unidas. 2000. Informe para la Asamblea de las Naciones Unidas dedicada al Milenio: "Nosotros los pueblos: la función de las Naciones Unidas en el siglo XXI”. Doc. A/54/2000.

Secretario General de las Naciones Unidas. 2005. Informe "Un concepto más amplio de libertad: desarrollo, seguridad y derechos humanos para todos". Doc. A/59/2005.

Secretario General de las Naciones Unidas. 2009. Informe "Hacer efectiva la responsabilidad de proteger". Doc. A/63/677.

TheAsia-Pacific Centre for the Responsibility to Protect. 2009. The Responsibility to Protect and the Protection of Civilians: Asia-Pacific in the UN Security Council. Update 1. Disponible en http://responsibilitytoprotect.org/files/ PoC_Update_1\%5B1\%5D\%5B1\%5D.pdf (accedido 28-2-14).

Tuck, Richard. 2002. The Rights of War and Peace: Political Thought and the International Order from Grotius to Kant. Oxford: Oxford University Press.

Uruguay. 2012. Intervención del Representante Permanente de Uruguay ante las Naciones Unidas, José Luis Cancela, en el Diálogo Temático de la Asamblea General sobre Responsabilidad de Proteger. Nueva York, 5 de septiembre de 2012.

Uruguay. 2013. Intervención del Representante Permanente de Uruguay ante las Naciones Unidas, José Luis Cancela, en el Diálogo Temático de la Asamblea General sobre Responsabilidad de Proteger. Nueva York, 11 de septiembre de 2013.

Venezuela. 2009. Intervención del Representante Permanente de Venezuela ante las Naciones Unidas, Jorge Valero, en el Diálogo Temático de la Asamblea General sobre Responsabilidad de Proteger. Nueva York, 24 de julio de 2009.

Venezuela. 2013. Intervención del Representante Permanente de Venezuela ante las Naciones Unidas, Samuel Moncada, en el Diálogo Temático de la Asamblea General sobre Responsabilidad de Proteger. Nueva York, 11 de julio de 2013.

World Federalist Movement and International Policy Group. 2003. "Civil society perspectives on the responsibility to protect, Final Report". 30 de abril de 2003. 